\title{
Quantitative determination of the nonlinear bulk and surface response from alpha-quartz using phase sensitive SFG spectroscopy
}

Cite as: J. Chem. Phys. 151, 064707 (2019); https://doi.org/10.1063/1.5109868

Submitted: 13 May 2019 . Accepted: 09 July 2019 . Published Online: 09 August 2019

Martin Thämer (D), Tobias Garling, R. Kramer Campen (D), and Martin Wolf

\section{COLLECTIONS}

This paper was selected as Featured
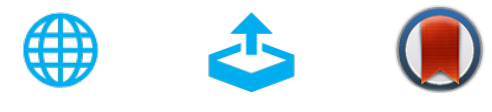

\section{ARTICLES YOU MAY BE INTERESTED IN}

Quadrupolar mechanism for vibrational sum frequency generation at air/liquid interfaces:

Theory and experiment

The Journal of Chemical Physics 151, 064701 (2019); https://doi.org/10.1063/1.5088192

Design of far-field thermal rectifiers using gold-vanadium dioxide micro-gratings Journal of Applied Physics 126, 063106 (2019); https://doi.org/10.1063/1.5100624

Generation of a microwave beam with both orbital and spin angular momenta using a transparent metasurface

Journal of Applied Physics 126, 063108 (2019); https://doi.org/10.1063/1.5109291

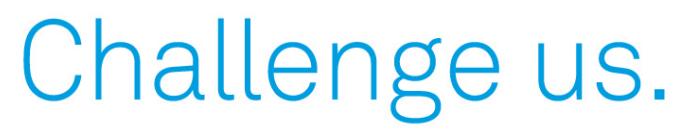

What are your needs for periodic signal detection?
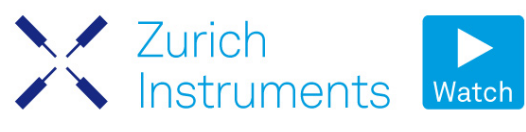

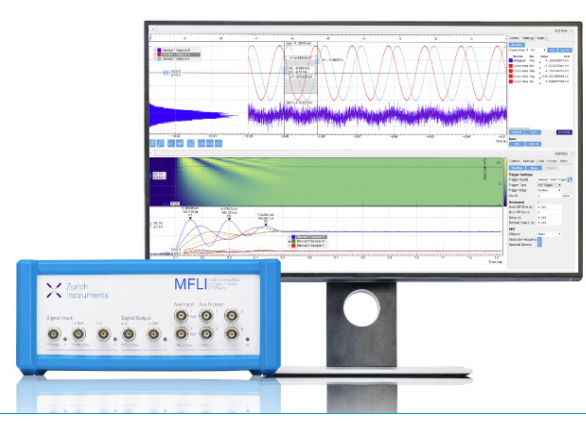

151, 064707 


\title{
Quantitative determination of the nonlinear bulk and surface response from alpha-quartz using phase sensitive SFG spectroscopy
}

\author{
Cite as: J. Chem. Phys. 151, 064707 (2019); doi: 10.1063/1.5109868 \\ Submitted: 13 May 2019 - Accepted: 9 July 2019 • \\ Published Online: 9 August 2019
}

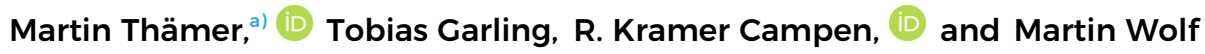

AFFILIATIONS

Fritz Haber Institute of the Max Planck Society, 4-6 Faradayweg, 14195 Berlin, Germany

a) Electronic mail: thaemer@fhi-berlin.mpg.de

\begin{abstract}
We investigate the nonlinear optical response of alpha-quartz with phase-sensitive vibrational sum frequency generation (SFG) spectroscopy. Phase and amplitude of the generated sum frequency signal are determined by a complex interplay between signal contributions from the surface and the bulk of the crystal with the experimental geometry, in particular, the quartz azimuth. By combining anisotropy measurements with spectral- and phase resolution in our SFG experiment, we study this interplay and show that we can quantitatively isolate and characterize the contributions from the surface and bulk of the crystal. The obtained optical constants in combination with the presented mathematical framework fully describe the nonlinear response and allow for a precise determination of the phase of the overall SFG signal of quartz for any experimental geometry. This knowledge is of particular relevance because quartz is commonly used as a reference in phase sensitive SFG experiments. In such studies, the surface contribution is typically neglected and a constant value for the phase of the sum frequency response is assumed. Our study shows that this assumption is not generally accurate by revealing the considerable impact that the surface contribution can have on the overall response of the crystal.
\end{abstract}

Published under license by AIP Publishing. https://doi.org/10.1063/1.5109868

\section{INTRODUCTION}

The physical properties of surfaces and interfaces play an essential role in wide fields of condensed matter physics, chemistry, and biology. The presence of an interface leads to the creation of surface bound electronic and vibrational states that can considerably differ from the corresponding bulk. The broad relevance of surface specific properties has triggered an enormous amount of research where surface sensitive experimental tools are typically employed. Various important processes that occur at solid surfaces, however, are not purely dominated by surface effects but also involve the coupling between surface and bulk states or the transport of charges or energy between bulk and surface. Prominent examples are electron relaxation in metals ${ }^{1,2}$ or charge transport in semiconductors, ${ }^{3}$ topological insulators, ${ }^{4,5}$ or photocatalysts. ${ }^{6}$ To investigate the details of these processes, it is necessary to study both the surface and the underlying bulk of a sample.

Performing such experimental bulk/surface studies is, however, challenging. To accurately investigate their interaction, surface and bulk of the sample should be probed with the same technique and ideally within one single experiment, while the analysis of transport processes additionally requires a high temporal resolution. One major difficulty of simultaneously probing surface and bulk of a material is to precisely extract their individual signals from an overall sample response. To perform such a decomposition, one needs to find a way to distinguish these two contributions which is far from trivial. It is in fact not always obvious how to define surface and bulk properties of a material because there is typically no clear boundary between a surface layer with a certain thickness and the bulk. Instead, the material properties typically show a smooth transition between these two limiting cases. Furthermore, signals from the bulk tend to be much stronger than signals from surfaces since they originate from a much larger volume. This makes it generally difficult to detect a surface contribution in the presence of a bulk signal and requires a large dynamic range of the experimental technique.

As we will show in the following, one experimental technique that can overcome these challenges is the laser based technique of 
phase-sensitive sum frequency generation spectroscopy (PS-SFG). SFG spectroscopy is typically applied to study interfaces; ${ }^{7-15}$ however, in case that the bulk of a sample lacks centrosymmetry both can be probed, its surface and the bulk. ${ }^{16,17}$ Like all second order nonlinear spectroscopic techniques, the generated (SFG) signal carries detailed information on the symmetry of the sample. This sensitivity to symmetry becomes apparent, e.g., in anisotropy measurements of the nonlinear response. ${ }^{18-21}$ The bulk and surface of any material differ intrinsically in their symmetry which results in different spectroscopic signatures in the SFG anisotropy data. Therefore, this property can be exploited in a symmetry analysis to distinguish the two contributions. Moreover, because of phase propagation effects, surface and bulk SFG signals contain characteristic relative phase shifts (see Sec. II). Combining phase resolution with SFG anisotropy measurements, a rich data-set is obtained allowing for a quantitative analysis of the different contributions to the overall signal.

In the SFG study presented here, we investigate the nonresonant sum frequency response from a z-cut alpha-quartz crystal. Using our recently developed time-domain, phase-sensitive, SFG spectrometer, ${ }^{22}$ we perform phase resolved anisotropy measurements and show that the nonlinear response from the crystal can quantitatively be dissected into bulk and surface contributions. Using this technique, both sample properties can be simultaneously characterized with an ultrafast probe offering femtosecond time resolution.

The interplay between surface and bulk contributions to the sum frequency signal from alpha-quartz has a particular relevance in the field of phase resolved SFG studies. An alpha-quartz crystal is here typically used as reference to calibrate phase and amplitude of the measured raw spectra. ${ }^{23-25}$ One of the key benefits of phasesensitivity in v-SFG studies is that instead of the ordinary magnitude spectra it yields the full complex vibrational spectra which contain valuable additional information (e.g., on molecular orientations). ${ }^{26-31}$ To obtain properly phased complex spectra, however, the intrinsic nonlinear response of the reference must be precisely known. Already small deviations between the assumed and the correct values can thereby have important consequences. For example, phase resolved spectra from the air-water interface exhibit small but significant differences when using different reference materials. These differences may originate from inaccuracies in the assumed reference phase and have considerable impact on the interpretation of the interfacial water structure. While the exact nature of the interfacial water layer is still heavily debated, these contradictory results have produced some ongoing controversy about appropriate referencing in PS-SFG spectroscopy and the exact phases of the nonresonant responses from different materials including alpha-quartz. ${ }^{32-34}$ This example shows that there is obviously a need for a more detailed investigation of the sum frequency response of alpha-quartz for its use as a reference.

It is, in general, challenging to theoretically predict the nonlinear optical response from a material even if the light matter interaction is fully nonresonant. This is, in particular, true for the phase of the sum frequency signal. Due to the intrinsic phase shift between the contributions from bulk and surface, the phase of the overall signal sensitively depends on their relative amplitudes. In the case of alpha-quartz, the nonlinear optical properties of the bulk material are well studied but little is known about its surface response. In fact, the surface contribution is typically assumed small enough to be neglected. Without a precise knowledge of the amplitude ratio of bulk and surface contributions, however, it is not clear if this assumption is justified. Furthermore, this ratio is not a fixed material property. Since surface and bulk differ in their symmetry, the relative amplitudes of the two contributions typically depend on the experimental arrangement such as crystal orientation, crystal cut, incidence angles, and polarizations of the laser beams (see Sec. II). The phase of the overall signal can therefore not be represented by a single value but is a result of a complex interplay between surface and bulk contributions and experimental geometry. With our PS-SFG study on alpha-quartz, we address exactly this complexity by studying the details of this interplay.

In Sec. II, we briefly review the basic theory of the sum frequency generation process followed by a theoretical analysis of the sum frequency response from alpha-quartz based on crystal symmetry and phase effects (Sec. III). We then present in Sec. $V$ the results from our phase-resolved anisotropy measurements and compare these data with theory. Based on this comparison, we are able to extract nonlinear optical constants for the bulk and the surface of the sample which fully describe the nonlinear optical properties of the quartz crystal. Finally, in Sec. VI, the impact of our results for the use of alpha-quartz as reference crystal in PS-SFG measurements is discussed.

\section{THEORY}

A generalized theory including a detailed mathematical description of the SFG process can be found elsewhere; ${ }^{35,36}$ here, we only revise some of the key elements. In the SFG spectroscopy, a sum frequency field $E_{S F G}$ is generated by nonlinear mixing of an infrared $E_{I R}$ and a visible laser field $E_{v i s}$ at the sample surface. All details of this frequency conversion process (phase and amplitude of the resulting SFG signal) are determined by the complex effective second order susceptibility $\chi_{e f f}^{(2)}$ of the sample which carries the desired spectroscopic information on vibrational resonances and molecular orientation

$$
E_{S F G}\left(\omega_{3}\right) \sim \chi_{\text {eff }}^{(2)}\left(\omega_{3}=\omega_{1}+\omega_{2}\right) \cdot E_{I R}\left(\omega_{1}\right) \cdot E_{v i s}\left(\omega_{2}\right) .
$$

The resulting SFG signal that is reflected from the sample surface is not necessarily generated in the surface region alone. In noncentrosymmetric media, the signal can originate from numerous atomic layers reaching several tens of nanometers into the bulk of the material (similar to the case of linear reflection). ${ }^{37}$ The overall reflected sum frequency signal is then a superposition of the contributions from all these layers. This can mathematically be expressed by integrating the different contributions along the surface normal ( $z$-coordinate; see Fig. 1), while properly accounting for the phase propagation effects. In case that the sample is nonabsorbing for all three frequencies $\left(\omega_{1}\right.$, $\omega_{2}$, and $\left.\omega_{3}\right)$, this integration can be expressed by following equation:

$$
\chi_{e f f}^{(2)}=\int_{-\infty}^{0} d z \cdot \chi_{z, e f f}^{(2)} \cdot e^{i \Delta k_{z} z},
$$




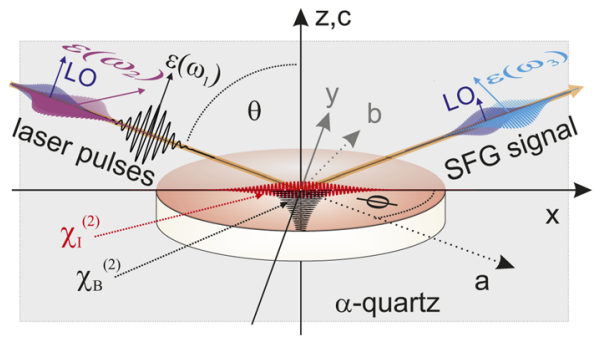

FIG. 1. Experimental arrangement for the phase resolved v-SFG measurements of $z$-cut quartz. $x, y, z$ are the Cartesian coordinates in the laboratory frame, and $a, b$, $c$ correspond to the crystallographic axes in the sample. $\phi$ is the azimuthal angle, and $\theta$ is the incidence angle of the laser pulses (plane of incidence is $x z$ ). Infrared (black) and $800 \mathrm{~nm}$ (purple) pulses generate the sum frequency signal (light blue), $\varepsilon\left(\omega_{i}\right)$ represent the normalized electric field vectors of the corresponding pulse. The polarization of the local oscillator pulse (LO, dark blue) is always orthogonal to the selected polarization of the SFG signal. The polarization combination in this figure corresponds to SSP.

with $\Delta k_{z}$ representing the mismatch of the wavevector components in the $z$-direction $\Delta k_{z}=k_{z}\left(\omega_{1}\right)+k_{z}\left(\omega_{2}\right)-k_{z}\left(\omega_{3}\right)$, while $\chi_{z, \text { eff }}^{(2)}$ describes the nonlinear optical properties of the material as a function of $z$. This local susceptibility includes the nonlinear optical properties in the interfacial region $\left(z \rightarrow 0^{-}\right)$and the properties of the bulk for larger negative values of $z$. As mentioned in the Introduction, it is difficult to define a specific value for $z$ where the surface layer ends and the bulk starts. It is therefore useful not to dissect $\chi_{z, \text { eff }}^{(2)}$ based on a spatial coordinate but to split the susceptibility into two overlapping components with different depth profiles. ${ }^{35,38}$ This is done by defining the interfacial part of the susceptibility as the deviation from the bulk properties in the interfacial region, while the bulk susceptibility $\chi_{B, e f f}^{(2)}$ describes the spatially homogeneous ( $z$-independent) part. Only the interfacial part is then a function of $z$ decaying to zero for larger negative values of $z$. Assuming an exponential decay of the interface term, the dissection of $\chi_{z, \text { eff }}^{(2)}$ can be expressed by the following equation:

$$
\chi_{z, e f f}^{(2)}=\chi_{I, e f f}^{(2)} \cdot e^{\frac{z}{z^{\prime}}}+\chi_{B, e f f}^{(2)},
$$

where $z^{\prime}$ is the characteristic length-scale on which deviation vanishes. It is important to note that the interface susceptibility $\chi_{I, e f f}^{(2)}$ in this notation does not represent the overall optical properties of the surface layer but their deviation from the bulk properties caused by the presence of the surface. It therefore purely describes the surface effect. Nevertheless, in the context of SFG spectroscopy, the part of the optical response arising from $\chi_{I, \text { eff }}^{(2)}$ and $\chi_{B, \text { eff }}^{(2)}$ is termed surface and bulk response, respectively. The clear advantage of this type of separation is that it offers an unambiguous definition of what part of the optical material properties relates to the surface properties. Combining Eqs. (2) and (3), the integral can be solved yielding

$$
\chi_{e f f}^{(2)}=\frac{\chi_{I, e f f}^{(2)}}{1 / z^{\prime}-i\left|\Delta k_{z}\right|}+\frac{\chi_{B, e f f}^{(2)}}{-i\left|\Delta k_{z}\right|} \text {. }
$$

Equation (4) shows how the surface and the bulk contribute to the overall sum frequency signal. Importantly, the terms describing the contribution from bulk and surface to $\chi_{\text {eff }}^{(2)}$ include phase shifts. The bulk contribution is shifted by exactly $90^{\circ}$ (caused by the imaginary unit in the denominator), whereas the phase shift of the surface part depends on the relative sizes of $z^{\prime}$ and $\left|\Delta k_{z}\right|$. In dielectric samples, it can usually be assumed that $z^{\prime} \ll 1 /\left|\Delta k_{z}\right|$ because the values for $1 /\left|\Delta k_{z}\right|$ are on the order of several tens of nanometers and the deviation should only extend into the first few atomic layers. The denominator of the surface term then becomes real (phase shift of $0^{\circ}$ ), and Eq. (4) can be simplified as

$$
\chi_{e f f}^{(2)} \approx \chi_{I, e f f}^{(2)} \cdot z^{\prime}+i \frac{\chi_{B, e f f}^{(2)}}{\left|\Delta k_{z}\right|} .
$$

Within this approximation, the contributions from surface and bulk to the overall susceptibility show a characteristic relative phase shift of exactly $90^{\circ}$. The phase of a sum frequency signal is therefore a good reporter on the origin of its source.

$\chi_{\text {eff }}^{(2)}$ describes the effective sum frequency response which depends on the experimental arrangement such as incidence angles and polarizations of the different laser pulses as well as the sample orientation. This dependency is accounted for by describing the nonlinear optical properties of the sample by the rank three susceptibility tensor $\chi_{G, x y z}^{(2)}$, where $x, y, z$ correspond to the Cartesian coordinates in the laboratory frame (see Fig. 1). This tensor has generally 27 elements but only a subset of these elements contributes to the sum frequency signal in a given experimental geometry. The particular contributions to $\chi_{\text {eff }}^{(2)}$ can be derived using following equation: ${ }^{39}$

$$
\begin{aligned}
\chi_{e f f}^{(2)}= & \sum_{i, j, k}\left[\vec{\varepsilon}\left(\omega_{3}\right) \cdot \vec{e}_{i} \cdot L_{i i}\left(\omega_{3}\right)\right] \cdot \chi_{G, i j k}^{(2)} \\
& \times\left[\vec{\varepsilon}\left(\omega_{2}\right) \cdot \vec{e}_{j} \cdot L_{j j}\left(\omega_{2}\right)\right]\left[\vec{\varepsilon}\left(\omega_{1}\right) \cdot \vec{e}_{k} \cdot L_{k k}\left(\omega_{1}\right)\right] .
\end{aligned}
$$

The different tensor elements are represented by the indices $i, j, k$ $=\{x, y, z\}, L_{i i}$ describes the $i$ th element of the transmission Fresnel coefficient matrix, and $\vec{e}_{i}$ is the unit vector in the $i$ th direction, whereas $\vec{\varepsilon}$ is the normalized electric field vector of the corresponding light pulse. Similar to Eq. (5), the overall response tensor $\chi_{G, x y z}^{(2)}$ can be expressed in terms of surface and bulk contributions

$$
\chi_{G, x y z}^{(2)}=\chi_{I, x y z}^{(2)} \cdot z^{\prime}+i \frac{\chi_{B, x y z}^{(2)}}{\left|\Delta k_{z}\right|} .
$$

The general form of the susceptibility tensor is related to the symmetry of the sample material. Depending on the corresponding point group, several of the 27 elements are zero and others are intrinsically related. ${ }^{40}$ The different symmetries of bulk and surface for any material then result in different forms of the tensors $\chi_{I, x y z}^{(2)}$ and $\chi_{B, x y z}^{(2)}$. This difference can be used as basis for their separation. The most simple example of such a separation by symmetry is the case of samples with a centro-symmetric bulk. In presence of centro-symmetry, all elements of the corresponding susceptibility tensor are zero under the dipole approximation. As a consequence, $\chi_{B, e f f}^{(2)}$ is highly suppressed and the signal is usually dominated by the surface response (at the surface, the centro-symmetry is broken). 
This is the typical case where v-SFG spectroscopy is applied and used as a surface specific tool. If the bulk symmetry is not centrosymmetric (such as alpha-quartz), however, the surface and bulk susceptibility tensors contribute with their specific elements to the overall response tensor. By analyzing the elements of $\chi_{G, x y z}^{(2)}$, the contributing elements from $\chi_{I, x y z}^{(2)}$ and $\chi_{B, x y z}^{(2)}$ can be identified by their characteristic positions within the response tensor and their different phase. This typically allows for a precise decomposition of the overall response tensor which permits to quantitatively recover the individual responses from bulk and surface.

To perform the described analysis of $\chi_{G, x y z}^{(2)}$, its different elements must be experimentally probed. There are several possibilities to vary the composition of tensor elements that contribute to $\chi_{\text {eff }}^{(2)}$; however, technically the most practical are (i) changing the polarizations of the incident laser beams and the detected sum frequency response and (ii) rotating the sample about the surface normal. Polarization changes correspond to rotations of the electric field vectors $\vec{\varepsilon}\left(\omega_{i}\right)$ in Eq. (6). There are in total 8 distinct polarization combinations possible for the three light pulses that are involved in the SFG process. The labeling convention for these cases is $P P P, P P S, P S P$, etc., with the polarization directions defined with respect to the plane of incidence as $S$ and $P$ and the order of the beams toward decreasing frequency $\left(\omega_{3}, \omega_{2}, \omega_{1}\right)$. The effect of the sample rotation on $\chi_{\text {eff }}^{(2)}$ can mathematically be described as an angular dependent coordinate transformation from the crystal frame $(a, b, c)$ into the laboratory frame $(x, y, z)$. In the case of a rotation about the surface normal, this transformation is given by

$$
\chi_{G, x y z}^{(2)}(\phi)=\sum_{a, b, c} R_{x a}^{z}(\phi) \cdot R_{y b}^{z}(\phi) \cdot R_{z c}^{z}(\phi) \cdot \chi_{G, a b c}^{(2)}
$$

with $a, b, c=\{x, y, z\} . R_{i j}^{z}$ corresponds to the $i j$ th element of the $3 \mathrm{D}$ rotation matrix for the rotation about the $\mathrm{z}$-axis and $\phi$ is the rotation angle. $\chi_{G, a b c}^{(2)}$ represents the overall nonlinear susceptibility tensor in coordinates of the crystal frame including bulk and surface parts. The phase-resolved SFG anisotropy measurement shown in this study is conducted by recording the complex spectrum while varying the azimuthal angle of the sample. This scan is subsequently repeated for different polarization combinations. Using Eqs. (6)-(8), the result of these measurements can be qualitatively predicted provided that the symmetries of bulk and surface of the investigated material are known.

\section{NONLINEAR RESPONSE FROM ALPHA-QUARTZ}

With this mathematical description in hand, we can turn to the theoretical analysis of the sum frequency response of alphaquartz. The bulk of alpha-quartz has $D_{3}$ symmetry, the nonvanishing elements of the bulk second order susceptibility are then $\chi_{B, a a a}^{(2)}$ $=-\chi_{B, a b b}^{(2)}=-\chi_{B, b b a}^{(2)}=-\chi_{B, b a b}^{(2)}, \chi_{B, a b c}^{(2)}=-\chi_{B, b a c}^{(2)}, \chi_{B, a c b}^{(2)}=-\chi_{B, b c a}^{(2)}$, and $\chi_{B, c a b}^{(2)}=-\chi_{B, c b a}^{(2)} \cdot{ }^{40}$ The determination of the symmetry related to the surface response is more challenging. As mentioned in Sec. II, $\chi_{I}^{(2)}$ does not describe the overall response from the surface layer but its deviation from the bulk. As a consequence, we cannot simply use the symmetry of the quartz surface to determine the nonvanishing tensor elements because it includes symmetry elements that relate to the bulk structure.

The second order susceptibility is directly related to the macroscopic potential asymmetry along a given polarization direction. The surface effects that modify the bulk potential landscape and thus give rise to a surface signal can be divided into three classes: (i) surface reconstruction (distortion of the crystal lattice near the surface), (ii) surface termination (formation of special terminal groups and adsorption of molecular species), and (iii) the macroscopic discontinuity in $\mathrm{z}$ (potential gradient across the interface induced by the discontinuity of the crystal). Among these three contributions, the effect of the discontinuity is most likely the dominant part. This assumption is supported by the good match between the theory and experiment which will be presented in this study. The symmetry of the discontinuity is $C_{\infty v}$, and the nonvanishing tensor elements in this point group are $\chi_{I, c c c}^{(2)}, \chi_{I, a a c}^{(2)}=\chi_{I, b b c}^{(2)}, \chi_{I, a c a}^{(2)}=\chi_{I, b c b}^{(2)}$, and $\chi_{I, c a a}^{(2)}=\chi_{I, c b b}^{(2)}$. Performing the coordinate transformation [Eq. (8)] on the bulk and the surface susceptibilities yields the angular dependent tensors $\chi_{i j k}^{(2)}(\phi)$ which can subsequently be inserted into Eq. (6) to calculate $\chi_{\text {eff }}^{(2)}$ for the four combinations of beam polarizations used in this study ( $P P P, S S P, S P P, P S P)$. The calculation yields following results:

$$
\begin{aligned}
\chi_{P P P, e f f}^{(2)}= & \cos (3 \phi) \cdot \frac{i}{\left|\Delta k_{z}\right|}\left[L_{x x}\left(\omega_{3}\right) L_{x x}\left(\omega_{2}\right) L_{x x}\left(\omega_{1}\right) \cos ^{3} \theta \cdot \chi_{B, a a a}^{(2)}\right] \\
& +\sin \theta \cdot z^{\prime}\left[L_{z z}\left(\omega_{3}\right) L_{z z}\left(\omega_{2}\right) L_{z z}\left(\omega_{1}\right) \sin ^{2} \theta \cdot \chi_{I, c c c}^{(2)}\right. \\
& -L_{x x}\left(\omega_{3}\right) L_{x x}\left(\omega_{2}\right) L_{z z}\left(\omega_{1}\right) \cos ^{2} \theta \cdot \chi_{I, a a c}^{(2)} \\
& -L_{x x}\left(\omega_{3}\right) L_{z z}\left(\omega_{2}\right) L_{x x}\left(\omega_{1}\right) \cos ^{2} \theta \cdot \chi_{I, a c a}^{(2)} \\
& \left.+L_{z z}\left(\omega_{3}\right) L_{x x}\left(\omega_{2}\right) L_{x x}\left(\omega_{1}\right) \cos ^{2} \theta \cdot \chi_{I, c a a}^{(2)}\right], \\
\chi_{S S P, e f f}^{(2)}= & -\cos (3 \phi) \cdot \frac{i}{\left|\Delta k_{z}\right|}\left[L_{y y}\left(\omega_{3}\right) L_{y y}\left(\omega_{2}\right) L_{x x}\left(\omega_{1}\right) \cos \theta \cdot \chi_{B, a a a}^{(2)}\right] \\
& -L_{y y}\left(\omega_{3}\right) L_{y y}\left(\omega_{2}\right) L_{z z}\left(\omega_{1}\right) \sin \theta \cdot z^{\prime} \cdot \chi_{I, a a c}^{(2)}, \\
\chi_{S P P, e f f}^{(2)}= & \sin (3 \phi) \cdot \frac{i}{\left|\Delta k_{z}\right|}\left[L_{y y}\left(\omega_{3}\right) L_{x x}\left(\omega_{2}\right) L_{x x}\left(\omega_{1}\right) \cos ^{2} \theta \cdot \chi_{B, a a a}^{(2)}\right] \\
& +\frac{i}{\left|\Delta k_{z}\right|} L_{y y}\left(\omega_{3}\right) \sin \theta \cos \theta\left[L_{x x}\left(\omega_{2}\right) L_{z z}\left(\omega_{1}\right) \cdot \chi_{B, a b c}^{(2)}\right. \\
& \left.+L_{z z}\left(\omega_{2}\right) L_{x x}\left(\omega_{1}\right) \cdot \chi_{B, a c b}^{(2)}\right], \\
\chi_{P S P, e f f}^{(2)}= & \sin (3 \phi) \cdot \frac{i}{\left|\Delta k_{z}\right|}\left[L_{x x}\left(\omega_{3}\right) L_{y y}\left(\omega_{2}\right) L_{x x}\left(\omega_{1}\right) \cos ^{2} \theta \cdot \chi_{B, a a a}^{(2)}\right] \\
& -\frac{i}{\left|\Delta k_{z}\right|} L_{y y}\left(\omega_{2}\right) \sin \theta \cos \theta\left[L_{x x}\left(\omega_{3}\right) L_{z z}\left(\omega_{1}\right) \cdot \chi_{B, a b c}^{(2)}\right. \\
& \left.+L_{z z}\left(\omega_{3}\right) L_{x x}\left(\omega_{1}\right) \cdot \chi_{B, c a b}^{(2)}\right] .
\end{aligned}
$$

Note that because of our collinear beam geometry (see Fig. 1), only one incidence angle $(\theta)$ appears in the equations. First, we concentrate on the azimuthal symmetry of the resulting $\chi_{\text {eff }}^{(2)}$. For all calculated polarization combinations, $\chi_{\text {eff }}^{(2)}$ shows contributions with 3 -fold symmetry $(\cos (3 \phi)$ and $\sin (3 \phi)$, respectively) as well as isotropic components. In the cases of $P P P$ and SSP polarizations, the 
isotropic component arises from the surface signal whereas the bulk signal only shows an isotropic contribution for SPP and PSP. The 3 -fold contribution meanwhile originates exclusively from the $\chi_{B, a a a}^{(2)}$ tensor element.

Equations (9)-(12) represent the different contributions to the sum frequency signals as a function of the polarization scheme. Based on symmetry considerations, we can additionally extract information about the relative sizes of the contributing tensor elements. In absence of dispersion, the indices of the susceptibility tensor can be freely permuted without a change in value. This rule is known as Kleinman symmetry and can be used to reduce the number of independent tensor elements. ${ }^{41}$ Applying Kleinman symmetry to the case of alpha-quartz, we find that the independent elements $\chi_{B, a b c}^{(2)}, \chi_{B, a c b}^{(2)}$, and $\chi_{B, c a b}^{(2)}$ are all equal and zero. They are therefore typically neglected. In reality, however, dispersion in a material is never zero, but in case that the SFG process takes place under nonresonant conditions (as in our experiment), the effect of dispersion is relatively small. These three elements can therefore be expected to be much smaller than $\chi_{B, a a a}^{(2)}$ which would be nonzero even if Kleinman symmetry applied. Furthermore, the dispersion is particularly small between $\omega_{2}$ and $\omega_{3}$ because their frequencies are close. The change in value when permuting the first two indices of the susceptibility tensor is consequently smaller than permutations which include the third index. Using these findings, we can approximate the relative sizes of the nonvanishing tensor elements to $\chi_{B, a a a}^{(2)} \gg \chi_{B, a c b}^{(2)} \approx \chi_{B, c a b}^{(2)}$ $>\chi_{B, a b c}^{(2)}$ and $\chi_{I, c a a}^{(2)} \approx \chi_{I, a c a}^{(2)}$. The same argumentation also holds for the values of the transmission Fresnel factors. We can therefore assume that $L_{i i}\left(\omega_{3}\right) \approx L_{i i}\left(\omega_{2}\right)$

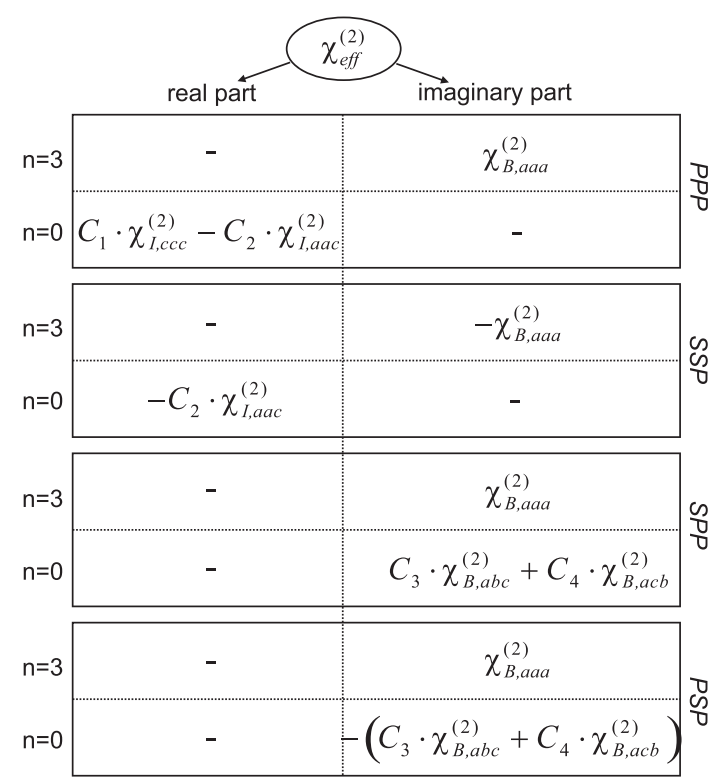

FIG. 2. Different tensor components from bulk and interface that contribute to the complex effective second order susceptibility of z-cut quartz. Contributions are classified into 3-fold $(n=3)$ and isotropic $(n=0)$ azimuthal symmetry and into real and imaginary parts. Each box (solid line) corresponds to a specific polarization scheme.
Finally, we analyze the phases of the different contributions. From Raman and FT-IR studies reported in the literature, we can conclude that there are no resonances in the spectrum of bulk quartz from the visible range through the midinfrared down to $\sim 1200 \mathrm{~cm}^{-1}$, where the first phonon resonances appear. ${ }^{42-44}$ The bulk nonlinear response is consequently nonresonant in the frequency ranges used in this study (see Sec. IV). The same conclusion should hold for the surface response since the surface phonons appear in the same lowfrequency range as the bulk phonons. ${ }^{39}$ The bulk and surface second order susceptibilities $\chi_{B, e f f}^{(2)}$ and $\chi_{I, e f f}^{(2)}$ must then be real with a flat spectral response. ${ }^{40}$ The interface contributions therefore appear in the real part of $\chi_{\text {eff }}^{(2)}$, whereas the bulk signal solely contributes to the imaginary part.

Combining all these considerations and approximations, the complexity of Eqs. (9)-(12) can greatly be reduced. The results are summarized in a compact form in the table in Fig. 2. For better clarity, all components are normalized to $\chi_{B, a a a}^{(2)}$ and the resulting prefactors of the susceptibilities are combined in the different $C_{i}$ (exact expressions for the $C_{i}$ are given in the supplementary material).

So far, we have only considered dipolar contributions to the signal. In principle, also signals with nonlocal quadrupolar origin could contribute to the spectra. ${ }^{4-47}$ Quadrupolar contributions to sum frequency signals are, in general, much smaller than the corresponding dipolar signals and are therefore commonly neglected. In the presented case, however, signals over a large range of amplitudes are studied and this approximation might not be justified. The nonlinear susceptibility that describes the quadrupolar contributions is formally of third order and consequently represented by a rank 4 tensor. $^{35,36}$ As a consequence, the quadrupolar signals can contribute quite differently to the overall $\chi_{\text {eff }}^{(2)}$ than the dipolar contributions. A qualitative comparison between theory (including quadrupolar contributions) and the results from our measurements is shown in the supplementary material. This analysis suggests that no significant contributions from quadrupolar bulk sources are present in our measured data which confirms our assumption that we can accurately describe the sum frequency response in the dipolar limit.

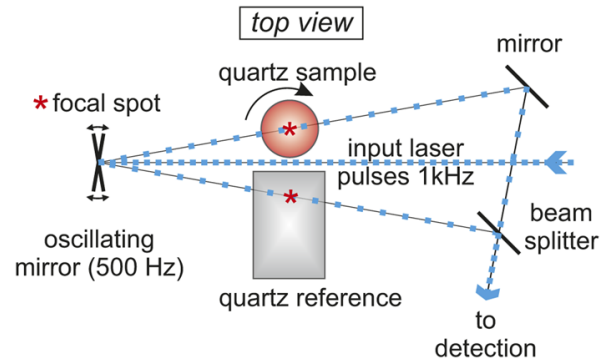

FIG. 3. Schematic illustration of the setup for shot to shot referencing. The sample quartz is rotated in steps of $6^{\circ}$ during the anisotropy measurement, while the reference quartz remains static. The interferogram from the reference is used to remove drifts in phase and amplitude from the sample data. 


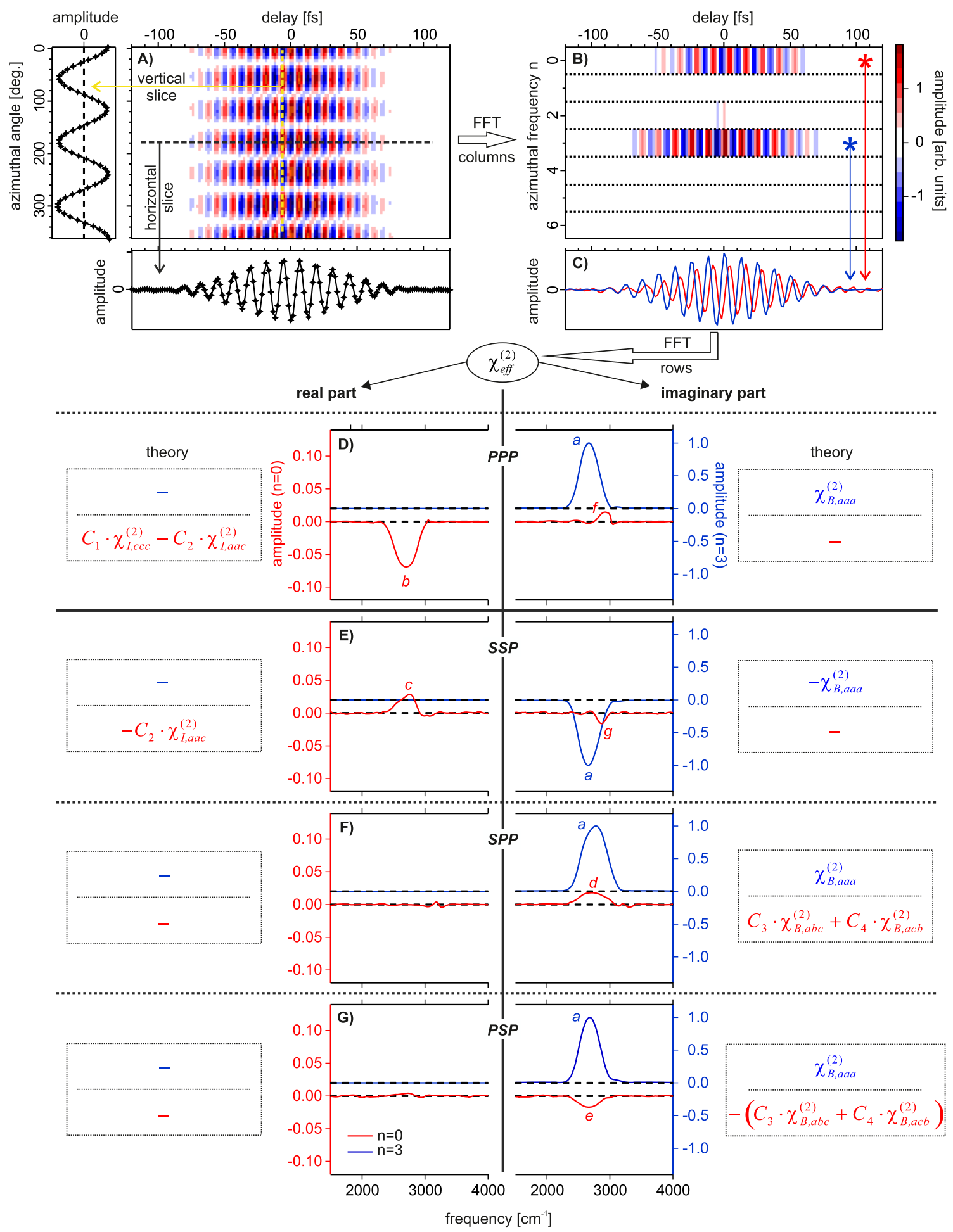

FIG. 4. Phase resolved SFG data from z-cut quartz. (a) background corrected raw data showing the time domain signal as a function of the azimuthal angle with horizontal and vertical slices; (b) real part after Fourier transformation along the vertical axis showing time domain signal as a function of azimuthal frequency; (c) representation of the isotropic (red line) and 3-fold (blue line) components [3-fold component is divided by 10 in (b) and (c)]; (d)-(g) resulting decomposed spectra after Fourier transformation along the horizontal axis for different polarization schemes. Blue and red lines correspond to 3 -fold and isotropic contribution, respectively, with the corresponding axes in the same color. 


\section{EXPERIMENTAL}

The v-SFG spectra are measured with our broadband, phase sensitive, time domain SFG spectrometer which is described in detail elsewhere. ${ }^{22} 800 \mathrm{~nm}\left(\omega_{2}\right)$ pulses are generated by an amplified Ti:Sa femtosecond laser system ( $1 \mathrm{kHz}$ repetition rate), and infrared pulses ( $\sim 10 \mu$ J pulse energy) are obtained by difference frequency mixing between the two outputs from an optical parametric amplifier. All measurements are carried out with the infrared pulses tuned to a center frequency of $2660 \mathrm{~cm}^{-1}$ and a bandwidth of $\sim 400 \mathrm{~cm}^{-1}$ (FWHM). The infrared beam is split into two parts (ratio 95:5), and the weak portion is combined with the $800 \mathrm{~nm}$ upconversion beam $(\sim 10 \mu \mathrm{J}$ per pulse) in a $50 \mu \mathrm{m}$ thick z-cut quartz window to generate a weak sum frequency signal serving as a local oscillator (LO). The LO and $800 \mathrm{~nm}$ pulses subsequently copropagate collinearly and pass a delay line with a computer-controlled translation stage. Both pulses are then collinearly combined with the main infrared portion and focused onto the sample surface at an incidence angle of $70^{\circ}$. generating the sample SFG signal. The interference between the local oscillator and the sample SFG is subsequently determined by detecting the pulse intensities using two avalanche photo diodes in a balanced detection scheme. By scanning the translation stage, both the local oscillator and the upconversion pulse are delayed with respect to the main infrared pulse probing phase and amplitude of the induced vibrational coherence.

High accuracy in phase and amplitude in the measured spectra is achieved by shot to shot referencing of the acquired data. An oscillating mirror mounted on a galvo motor is installed in the setup which alternately samples $(500 \mathrm{~Hz})$ one spot on the alpha-quartz sample and another on a stationary quartz reference (see Fig. 3). Every time delay scan thus yields two interferograms: the responses from the sample and reference crystal. The reference interferogram is used in a fully automized procedure for prephasing of the sample spectra and to correct for changes in the SFG amplitude from scan to scan. This technique allows for efficient cancellation of the long-time phase and amplitude drifts over the course of the experiment. $^{22}$ Furthermore, since the orientation of the crystallographic axes inside the reference crystal is known, we can deduce the sign of its dominating bulk response for each polarization combination. This knowledge is used for adjusting the sample quartz to the correct starting position for the azimuthal scans. The quartz sample is placed on a computer-controlled rotation stage. A full azimuthal measurement is performed by rotating the quartz sample in steps of $6^{\circ}$ about its $\mathrm{z}$-axis while recording an entire interferogram of the sample. The result of such a measurement is a 2-dimensional "raw" data matrix which shows the amplitude of the interference as a function of both, the time delay and the azimuthal angle [see, e.g., Fig. 4(a)].

The measurements of the different polarization combinations are performed by changing the polarization of the incident beams. In contrast, the polarization of the detected sum frequency signal from the sample is controlled by tuning the polarization of the local oscillator which acts similar to a regular polarizer (see Ref. 22). Optically polished, z-cut alpha-quartz crystals (MaTeck) are used as a sample and a reference. Before each experiment, the top surface of the crystal was cleaned with lens cleaning tissue and a drop of spectroscopy grade acetone.
Additional information on experimental details can be found in the supplementary material.

\section{RESULTS}

Figure 4(a) shows the reference corrected raw data from the anisotropy scan of the sample quartz for the $P P P$ polarization combination. For better illustration of the two dimensional data, representative horizontal and vertical slices through the data matrix are included (every black cross in the slices corresponds to one data point). As shown in Sec. III, for $\chi_{P P P, e f f}^{(2)}$, we expect the presence of one component with 3 -fold and another with isotropic azimuthal symmetry. These different contributions can be extracted by performing a Fourier analysis. The raw data matrix is therefore Fourier transformed along the vertical axis, and after proper phasing (see the supplementary material), the real part of the resulting complex matrix [Fig. 4(b)] shows the different portions of the interferogram as a function of the azimuthal frequency $n(n=0$ corresponds to isotropic $n=1$ to 1 -fold symmetry, etc.). Figure 4 (b) shows that the measured raw data indeed consists of a superposition of the two predicted components. In Fig. 4(c), these two components are plotted on a common axis [for better clarity, the 3-fold component is divided by 10 in Figs. 4(b) and 4(c)]. In a final step, the two interferograms $(n=0$ and $n=3)$ are Fourier transformed (along the horizontal axis) into the frequency domain [Fig. 4(d)]. The final phasing of the resulting spectra is based on the spectrum of the 3-fold component. Following the assumption that the nonlinear bulk response is fully off-resonant and shifted by $90^{\circ}$ as a result of integrating its contributions along the $\mathrm{z}$ coordinate, the spectra are phased such that the 3-fold component purely appears in the imaginary part of the spectrum. Finally, all amplitudes are divided by the maximum absolute value of the 3 -fold component for normalization. This analysis is subsequently repeated for the measurement of the polarization combinations SSP, SPP, and PSP with the results depicted in Figs. 4(e), 4(f), and 4(g), respectively. These final spectra are the frequency resolved, complex $\chi_{\text {eff }}^{(2)}$ decomposed into their real and imaginary parts (left and right sides of the spectra) and into their isotropic and 3-fold azimuthal contributions (blue and red lines, respectively). For convenience and to enable a direct comparison between the experiment and theory, these theoretical results are added in Figs. $4(\mathrm{e})-4(\mathrm{~g})$ at the corresponding positions.

This comparison shows that all contributions predicted by the theoretical calculations appear in the measured spectra and are located in the predicted parts of the plot. The peaks labeled " $a$ " in Figs. $4(\mathrm{~d})-4(\mathrm{~g})$ obviously correspond to the $\chi_{B, a a a}^{(2)}$ tensor element of the bulk nonlinear susceptibility and appear in the imaginary part of $\chi_{e f f}^{(2)}$. Peaks $b$ and $c$ belong to the components $C_{1} \cdot \chi_{I, c c c}^{(2)}$ $-C_{2} \cdot \chi_{I, a a c}^{(2)}$ and $-C_{2} \cdot \chi_{I, a a c}^{(2)}$, respectively, and originate from the surface. In good agreement with theory, these surface contributions are found in the real part of $\chi_{\text {eff }}^{(2)}$ confirming the $90^{\circ}$ phase shift between bulk and surface signals. The surface response has, meanwhile, a significant size; in the $P P P$ polarization combination, its amplitude amounts to about $7 \%$ of the amplitude of the response from the dominating $\chi_{B, a a a}^{(2)}$ contribution. Furthermore, even the bulk 
components $C_{3} \cdot \chi_{B, a b c}^{(2)}+C_{4} \cdot \chi_{B, a c b}^{(2)}$ and $-\left(C_{3} \cdot \chi_{B, a b c}^{(2)}+C_{4} \cdot \chi_{B, a c b}^{(2)}\right)$ (peaks $d$ and $e$, respectively) are clearly resolved although they show the expected very small amplitude (roughly 60 times smaller than $\left.\chi_{B, a a a}^{(2)}\right)$. The observation of these two peaks in the spectra is only possible because Kleinman symmetry does not strictly apply in the presence of dispersion. Their presence is moreover a direct consequence of the chiral nature of alpha quartz. Tensor components of the form $\chi_{(i \neq j \neq k)}^{(2)}$ are exclusively nonzero in chiral systems and are therefore a good reporter of chirality. ${ }^{48}$ Also in perfect agreement with the theoretical results is the fact that $d$ and $e$ have similar amplitudes but appear in the corresponding spectra with opposite signs.

The only deviation from the theoretical results is the presence of the small spectral features, peaks $f$ and $g$, in the imaginary parts of $\chi_{P P P, e f f}^{(2)}$ and $\chi_{S S P, e f f}^{(2)}$ where theory predicts no signal. A closer look at these peaks shows that they have a much smaller bandwidth and a clearly shifted center frequency compared to all other observed peaks. These effects become more visible in Fig. 5 where the different peaks are magnified [solid lines in Figs. 5(a) and 5(b)]. For nonresonant SFG signals, the center frequency and spectral bandwidth are mainly given by the center frequency and bandwidth of the infrared pulses [see Eq. (1)]. All nonresonant contributions should therefore be spectrally similar which is indeed the case for all observed peaks except $f$ and $g$. This suggests that these two

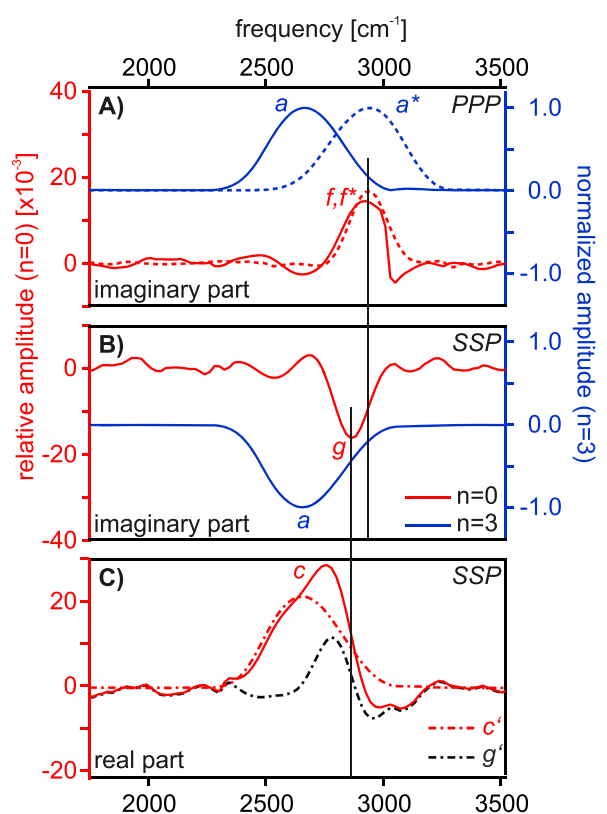

FIG. 5. Analysis of the resonant spectral contributions. Blue lines correspond to the 3 -fold and red lines correspond to the isotropic contributions with the corresponding axes in the same color. (a) and (b) show the imaginary parts of the effective susceptibility for the PPP and SSP polarization schemes, respectively. Dotted lines are the results of measurements at the shifted infrared center frequency. (c) shows the real part of the effective susceptibility with decomposition of the isotropic component into resonant (black dotted line) and nonresonant contributions (red dotted line). peaks have resonant character. This conclusion can be verified by shifting the center frequency of the infrared pulses. If $f$ and $g$ are indeed of resonant origin, their peak frequencies should not change. The entire SFG measurement for the $P P P$ polarization combination is therefore repeated with the infrared center frequency tuned to $2950 \mathrm{~cm}^{-1}$. After the decomposition the imaginary part is extracted and included in Fig. 5(a) (dotted lines, 3-fold component $a^{*}$ and isotropic component $\left.f^{*}\right)$.

The result of this measurement shows that the (nonresonant) 3 -fold component blue shifts together with the infrared frequency $\left(a \rightarrow a^{*}\right)$, whereas the isotropic component indeed stays at the same position $\left(f \rightarrow f^{*}\right)$. This clearly confirms that peaks $f$ and $g$ originate from a resonant contribution.

Comparing Figs. 5(a) and 5(b) shows that $g$ and $f$ do not peak at the same frequency but are slightly shifted. $f$ has its maximum at $\sim 2870 \mathrm{~cm}^{-1}$, whereas $g$ peaks at about $2940 \mathrm{~cm}^{-1}$. These are characteristic frequencies for the symmetric and asymmetric stretch vibrations of $\mathrm{CH}_{3} / \mathrm{CH}_{2}$ groups in organic compounds. ${ }^{12}$ The spectral differences between $g$ and $f$ can be explained by the fact that the symmetric stretch vibration of organic compounds on dielectric materials typically dominate in the SSP polarization scheme, whereas $P P P$ mainly samples the asymmetric stretch vibrations. ${ }^{49}$ We can therefore conclude that the observed resonant signals originate from a small contamination of the sample with hydrocarbons.

Resonant signals always generate complex spectra with contributions in both real and imaginary parts. There should consequently be counter parts to these resonant peaks in the real parts of the corresponding $\chi_{\text {eff }}^{(2)}$ mixed with the nonresonant surface signals $b$ and $c$. Taking the peak $c$ as example, we show that this is indeed the case. A closer look on the lineshape of $c$ shows that it is slightly distorted. As shown in Fig. 5(c), we can decompose $c$ into the two contributions $c^{\prime}$ and $g^{\prime}$, where $c^{\prime}$ matches the lineshape of the other nonresonant contributions. $g^{\prime}$ shows meanwhile a bipolar lineshape with its inflection point located at the peak position of $g$ (visualized by the vertical black line in the figure). This is exactly the expected spectral profile for the dispersive (real) counterpart of $g$. This analysis thus confirms that the corresponding real parts of the signals $g$ and $f$ are indeed present but buried under the nonresonant interface signal.

From the spectra in Fig. 4, we can learn even more about the sample contamination. The fact that the absorptive (imaginary) parts of the resonant signal appear in the imaginary parts of $\chi_{e f f}^{(2)}$ means that the contamination must be located at the surface and not in the bulk (as expected for a crystal contamination with hydrocarbons). Furthermore, the magnitude of the resonance signal is about 5 times smaller than the nonresonant surface contribution $b$. Considering that resonant SFG signals are generally much larger than nonresonant contributions and that $b$ originates from only the topmost atomic layers of the sample, it is reasonable to assume that the surface coverage of the organic contamination corresponds to only a small fraction of a monolayer. An in-depth analysis of the contamination would require measurements at higher frequency resolution (longer interferograms); however, this is beyond the scope of this study.

At this point, we have successfully assigned all spectral features that we observe in the decomposed spectra to specific 
contributions. We have separated different tensor elements of the bulk response and have isolated the signal from the surface. Furthermore, we have found a small resonant contribution that originates from a small contamination of the sample surface with organic compounds. All these spectral components cover a large range of amplitudes. The smallest recorded signals are the resonant peaks from the surface contamination which have an amplitude as small as $1.5 \%$ of the dominating 3 -fold bulk contribution. Despite their small size, they are still clearly resolved. The inaccuracy of our experiment can be estimated by measuring the deviations of the spectra from zero in those parts of the result where no assigned signals appear [e.g., the isotopic real parts in Figs. 4(f) and 4(g)]. The deviations there are on the order of $0.3 \%$ of the amplitude of peak $a$. This means that we cover with our spectrometer a dynamic range of $>100$. Note that the measured quantity in our experiments is the amplitude of the SFG field, while more commonly used homodyned SFG spectrometer measures the SFG intensity. The dynamic range that we achieve here thus corresponds to more than four orders of magnitude in intensity. This remarkable performance of our spectrometer owes to the combination of balanced heterodyne detection with the high accuracy in phase and amplitude. Our results clearly demonstrate that this large dynamic range is highly beneficial in SFG studies because it allows us to analyze very weak spectral features in the presence of comparatively large, dominating signals.

\section{DISCUSSION}

As shown in Sec. V, the results from the theory and experiment are in excellent agreement which confirms the accuracy of the presented theoretical considerations. The amplitudes of the different contributions obtained from the measurements in combination with the mathematical framework given in the theory sections now allow to unravel the details of the sum frequency response from alpha-quartz and its dependence on experimental geometry. The main impact of the interplay between bulk and surface responses on the overall SFG signal is the variation of its phase. As shown by the theory and experiment, the two contributions intrinsically differ in their phase by $90^{\circ}$ for nonabsorbing media and their relative sizes depend on the azimuthal angle and the polarization scheme. As a consequence, the phase of the emitted SFG signal also depends on these parameters. This behavior is quantitatively illustrated in Fig. 6 showing the phase and amplitude of the overall SFG signal as a function of the azimuthal angle and polarization combination. The calculation of the presented curves is performed by inserting the experimentally obtained values into Eqs. (9)-(12). Despite the relatively small size of the surface contribution ( $7 \%$ and $2 \%$ of the bulk contributions for $P P P$ and SSP, respectively), its impact on the overall phase can be clearly observed. For the $P P P$ case (red line), we see that the overall phase significantly deviates from the phase of the bulk contribution of $\pm 90^{\circ}$. The smallest deviation can be observed where the 3 -fold bulk contribution is maximized (at the azimuthal angles $0^{\circ}, 60^{\circ}$, etc.). The overall phase here has values of $\pm 94^{\circ}$; however, when turning the azimuthal angle away from these positions, the phase quickly increases toward $180^{\circ}(-180)^{\circ}$. For SSP, we observe the opposite trend. The smallest deviation yields a phase of $\pm 88.9^{\circ}$ (at $\phi=60^{\circ}$ ) which crosses the value of $0^{\circ}$ where the bulk contribution vanishes. In contrast to this angular dependent phase deviations, we obtain a

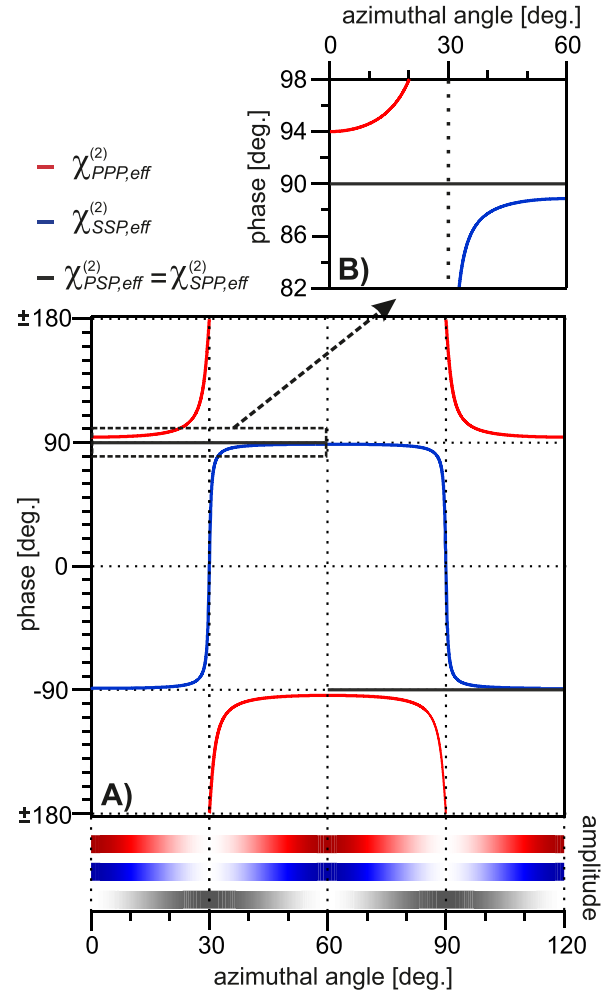

FIG. 6. (a) Phases of the overall SFG signal from z-cut quartz as a function of the azimuthal angle for different polarization schemes. Red line: PPP, blue line: SSP, and black line: SPP and PSP. Shaded color bars in the bottom part represent the corresponding amplitude of the emitted SFG field (absolute values). (b) Magnification of the marked area.

phase of exactly $\pm 90^{\circ}$ for the SPP and PSP polarization combinations (black line) because here the 3 -fold and the isotropic contributions both originate from the bulk and thus only contribute to the imaginary part of $\chi_{\text {eff }}^{(2)}$.

These large variations in phase shown in Fig. 6 report on the rich interplay between bulk and surface signals with the experimental geometry and characterize the overall sum frequency response of alpha-quartz in our experiment. In order to transform our measured quantities into optical constants of the alpha-quartz crystal, the prefactors $C_{i}$ are calculated and the absolute values for the different tensor elements of surface and bulk contributions are determined (for details, see the supplementary material). As reference quantity serves the bulk tensor component $\chi_{B, a a a}^{(2)}$ which has experimentally been determined by several studies. The resulting values are

$$
\begin{aligned}
& \chi_{B, a a a}^{(2)}=0.8 \mathrm{pm} / \mathrm{V}(\text { Ref. } 50), \\
& \chi_{B, a c b}^{(2)} \approx \chi_{B, c a b}^{(2)}=0.0079 \mathrm{pm} / \mathrm{V}, \\
& \chi_{I, c c c}^{(2)} \cdot z^{\prime}=-5.2 \times 10^{-22} \mathrm{~m}^{2} / \mathrm{V}, \\
& \chi_{I, a a c}^{(2)} \cdot z^{\prime}=-3.8 \times 10^{-22} \mathrm{~m}^{2} / \mathrm{V} .
\end{aligned}
$$


However, to properly compare the sizes of the surface and bulk susceptibilities, we need to know $z^{\prime}$ which describes the characteristic depth on which the nonlinear properties approach the bulk values. The quantity $z^{\prime}$ is an intrinsic material property and can in the case of quartz be estimated to be on the order of $z^{\prime}=1 \mathrm{~nm}$. ${ }^{34}$ This corresponds to a situation where the first 10 atomic layers would contribute to the surface signal. Using this value, we obtain for the two contributing surface susceptibilities $\chi_{I, c c c}^{(2)}=-0.52 \mathrm{pm} / \mathrm{V}$ and $\chi_{I, a a c}^{(2)}=-0.38 \mathrm{pm} / \mathrm{V}$. Interestingly, these resulting values have a very similar size as $\chi_{B, a a a}^{(2)}$ but the opposite sign. The fact that the contribution from the surface is much weaker than the bulk signal is therefore mainly related to the different sample volumes from which they are emitted $\left(z^{\prime} \ll 1 /\left|\Delta k_{z}\right|\right)$. Based on these values, the phase of the sum frequency response can be calculated for any experimental geometry.

What do we learn from these results for the use of alpha-quartz as a phase reference in PS-SFG studies? It is common to neglect the surface contribution and to assume a constant phase value of $\pm 90^{\circ}$ (phase of the bulk contribution) for the overall sum frequency response. ${ }^{34}$ Our results demonstrate, however, that the surface contribution is not as small as often believed. It can indeed have a considerable impact on the phase of the nonlinear response and should thus be accounted for. This is, in particular, true if the quartz reference is buried under some other material. Since the magnitude of the surface response strongly depends on the potential gradient at the interface, it can be expected to sensitively react to changes in its dielectric environment. In such cases, the surface response could easily be even larger than in our study. This sensitivity can on the other hand be exploited to obtain insight into the electrical properties of the interface between quartz and, e.g., various liquids by performing similar experiments as shown here on such systems. Furthermore, our study shows the presence of a contamination of the quartz surface with hydrocarbons. While it is possible that the detected hydrocarbons originate from the described cleaning of the surface, such contamination is likely a common phenomenon. Even if the effect of these undesired signals on the phase and amplitude is small, their rather narrow spectral profile can potentially introduce small peaks in a sample spectrum when the quartz spectrum is used as a reference. Appropriate cleaning steps might be required to reduce this problem.

\section{SUMMARY}

In this paper, we have investigated the details of the nonlinear optical response from $\mathrm{z}$-cut alpha-quartz using phase resolved sum frequency generation spectroscopy. The decomposition of the overall signal separates the contributions from different tensor elements and isolates the surface signal. To our knowledge, the resulting optical constants have not yet been quantitatively determined in an SFG experiment. The success of the decomposition is based on the combination of anisotropy measurements with phase and spectral resolution in one SFG experiment. The large dynamic range of $>100$ that we achieve with our instrument allows us to resolve even the weak signals arising from tensor elements which are zero under Kleinman approximation and to identify and characterize small contaminations of the sample surface with organic compounds.
As a result of our measurements, we find that the relative size of the surface contribution is indeed significant and that it can have a considerable impact on the phase of the overall sum frequency response of alpha-quartz. Besides the utility of our results for the use of alpha-quartz as a phase reference, the presented results demonstrate more generally how we can obtain detailed spectroscopic information about surface and bulk properties of materials within one experiment. An interesting extension of the presented study would be to tune to lower infrared frequencies and probe the bulk and surface phonons in quartz. Applying the presented technique in combination with selectively pumping different parts of the phonon spectrum would then allow for investigating the coupling of bulk and surface phonons and to follow the energy flow between these different vibrational modes of the crystal.

\section{SUPPLEMENTARY MATERIAL}

See supplementary material for details on the calculation of the susceptibilities, the determination of the background contributions, details of the data analysis, and a discussion of possible signal contributions from quadrupole transitions.

\section{ACKNOWLEDGMENTS}

This study was supported by the European Research Council (ERC) under the European Union's Horizon 2020 research and innovation program (Grant No. 772286 to R.K.C.). The authors thank Yujin Tong for intense discussions and help with the theoretical description of the results.

\section{REFERENCES}

${ }^{1}$ J. Kliewer, R. Berndt, E. V. Chulkov, V. M. Silkin, P. M. Echenique, and S. Crampin, "Dimensionality effects in the lifetime of surface states," Science $\mathbf{2 8 8}$, 1399-1402 (2000).

${ }^{2}$ A. Eiguren, B. Hellsing, F. Reinert, G. Nicolay, E. V. Chulkov, V. M. Silkin, S. Hüfner, and P. M. Echenique, "Role of bulk and surface phonons in the decay of metal surface states," Phys. Rev. Lett. 88, 066805 (2002).

${ }^{3}$ W. A. Tisdale, K. J. Williams, B. A. Timp, D. J. Norris, E. S. Aydil, and X.-Y. Zhu, "Hot-electron transfer from semiconductor nanocrystals," Science 328, 1543-1547 (2010).

${ }^{4}$ J. A. Sobota, S.-L. Yang, D. Leuenberger, A. F. Kemper, J. G. Analytis, I. R. Fisher, P. S. Kirchmann, T. P. Devereaux, and Z.-X. Shen, "Distinguishing bulk and surface electron-phonon coupling in the topological insulator $\mathrm{Bi}_{2} \mathrm{Se}_{3}$ using time-resolved photoemission spectroscopy," Phys. Rev. Lett. 113, 157401 (2014).

${ }^{\mathbf{5}}$ H. Steinberg, J.-B. Laloë, V. Fatemi, J. S. Moodera, and P. Jarillo-Herrero, "Electrically tunable surface-to-bulk coherent coupling in topological insulator thin films," Phys. Rev. B 84, 233101 (2011)

${ }^{6}$ A. L. Linsebigler, G. Lu, and J. T. Yates, "Photocatalysis on $\mathrm{TiO}_{2}$ surfaces: Principles, mechanisms, and selected results," Chem. Rev. 95, 735-758 (1995).

${ }^{7}$ Y. R. Shen, "Surface properties probed by second-harmonic and sum-frequency generation," Nature 337, 519-525 (1989).

${ }^{8}$ D. Zhang, J. Gutow, and K. B. Eisenthal, "Vibrational spectra, orientations, and phase transitions in long-chain amphiphiles at the air/water interface: Probing the head and tail groups by sum frequency generation," J. Phys. Chem. 98, 1372913734 (1994).

${ }^{9}$ C. D. Bain, "Sum-frequency vibrational spectroscopy of the solid-liquid interface," J. Chem. Soc. Faraday Trans. 91, 1281-1296 (1995).

${ }^{10}$ M. Bonn, C. Hess, S. Funk, J. H. Miners, B. N. J. Persson, M. Wolf, and G. Ertl, "Femtosecond surface vibrational spectroscopy of $\mathrm{CO}$ adsorbed on $\mathrm{Ru}(001)$ during desorption," Phys. Rev. Lett. 84, 4653-4656 (2000). 
${ }^{11}$ G. L. Richmond, "Molecular bonding and interactions at aqueous surfaces as probed by vibrational sum frequency spectroscopy," Chem. Rev. 102, 2693-2724 (2002).

${ }^{12}$ Z. Chen, Y. R. Shen, and G. A. Somorjai, "Studies of polymer surfaces by sum frequency generation vibrational spectroscopy," Annu. Rev. Phys. Chem. 53, 437465 (2002).

${ }^{13}$ M. Sovago, R. K. Campen, H. J. Bakker, and M. Bonn, "Hydrogen bonding strength of interfacial water determined with surface sum-frequency generation," Chem. Phys. Lett. 470, 7-12 (2009).

${ }^{14}$ P. B. Davies, "Interference effects in the sum frequency generation spectra of thin organic films. II: Applications to different thin-film systems," J. Chem. Phys. 133, 034705 (2010).

${ }^{15}$ C. S. Tian and Y. R. Shen, "Recent progress on sum-frequency spectroscopy," Surf. Sci. Rep. 69, 105-131 (2014).

${ }^{16}$ V. Ostroverkhov, G. A. Waychunas, and Y. R. Shen, "New information on water interfacial structure revealed by phase-sensitive surface spectroscopy," Phys. Rev. Lett. 94, 046102 (2005).

${ }^{17}$ W.-T. Liu and Y. R. Shen, "Sum-frequency spectroscopy on bulk and surface phonons of noncentrosymmetric crystals," Ann. Phys. 523, 101-106 (2011).

${ }^{18}$ S. Malyk, F. Y. Shalhout, L. E. O’Leary, N. S. Lewis, and A. V. Benderskii, "Vibrational sum frequency spectroscopic investigation of the azimuthal anisotropy and rotational dynamics of methyl-terminated silicon(111) surfaces," J. Phys. Chem. C 117, 935-944 (2013)

${ }^{19}$ S. Nihonyanagi, D. Miyamoto, S. Idojiri, and K. Uosaki, "Evidence for epitaxial arrangement and high conformational order of an organic monolayer on $\mathrm{Si}(111)$ by sum frequency generation spectroscopy," J. Am. Chem. Soc. 126, 7034-7040 (2004).

${ }^{20} \mathrm{C}$. Bilger and B. Pettinger, "The SHG anisotropy pattern of a reconstructed gold(110) electrode,” Chem. Phys. Lett. 294, 425-433 (1998).

${ }^{21} \mathrm{C}$. Yamada and T. Kimura, "Anisotropy in second-harmonic generation from reconstructed surfaces of GaAs,” Phys. Rev. Lett. 70, 2344-2347 (1993).

${ }^{22}$ M. Thämer, R. K. Campen, and M. Wolf, "Detecting weak signals from interfaces by high accuracy phase-resolved sfg spectroscopy," Phys. Chem. Chem. Phys. 20, 25875-25882 (2018).

${ }^{23}$ D. K. Hore, M. Y. Hamamoto, and G. L. Richmond, "Mid-infrared second-order susceptibility of $\alpha$-quartz and its application to visibile-infrared surface sum-frequency spectroscopy," J. Chem. Phys. 121, 12589-12594 (2004).

${ }^{24}$ R. Superfine, J. Huang, and Y. Shen, "Experimental determination of the sign of molecular dipole moment derivatives: An infrared-visible sum frequency generation absolute phase measurement study," Chem. Phys. Lett. 172, 303-306 (1990).

${ }^{25}$ X. Wei, S.-C. Hong, X. Zhuang, T. Goto, and Y. R. Shen, "Nonlinear optical studies of liquid crystal alignment on a rubbed polyvinyl alcohol surface," Phys. Rev. E 62, 5160-5172 (2000).

${ }^{26}$ R. Superfine, J. Y. Huang, and Y. R. Shen, "Phase measurement for surface infrared-visible sum-frequency generation," Opt. Lett. 15, 1276-1278 (1990).

${ }^{27}$ N. Ji, V. Ostroverkhov, C.-Y. Chen, and Y.-R. Shen, "Phase-sensitive sumfrequency vibrational spectroscopy and its application to studies of interfacial alkyl chains," J. Am. Chem. Soc. 129, 10056-10057 (2007).

${ }^{28}$ I. V. Stiopkin, H. D. Jayathilake, A. N. Bordenyuk, and A. V. Benderskii, "Heterodyne-detected vibrational sum frequency generation spectroscopy," J. Am. Chem. Soc. 130, 2271-2275 (2008).
${ }^{29}$ S. Yamaguchi and T. Tahara, "Heterodyne-detected electronic sum frequency generation: 'up' versus ‘down' alignment of interfacial molecules,” J. Chem. Phys. 129, 101102 (2008).

${ }^{30}$ S. Nihonyanagi, T. Ishiyama, T.-k. Lee, S. Yamaguchi, M. Bonn, A. Morita, and T. Tahara, "Unified molecular view of the air/water interface based on experimental and theoretical $\chi^{(2)}$ spectra of an isotopically diluted water surface," J. Am. Chem. Soc. 133, 16875-16880 (2011).

${ }^{31}$ Y. Shen, "Phase-sensitive sum-frequency spectroscopy," Annu. Rev. Phys. Chem. 64, 129-150 (2013).

${ }^{32}$ S. Nihonyanagi, R. Kusaka, K.-i. Inoue, A. Adhikari, S. Yamaguchi, and T. Tahara, "Accurate determination of complex $\chi(2)$ spectrum of the air/water interface,” J. Chem. Phys. 143, 124707 (2015).

${ }^{33}$ S. Yamaguchi, "Development of single-channel heterodyne-detected sum frequency generation spectroscopy and its application to the water/vapor interface," J. Chem. Phys. 143, 034202 (2015).

${ }^{34}$ S. Sun, R. Liang, X. Xu, H. Zhu, Y. R. Shen, and C. Tian, "Phase reference in phase-sensitive sum-frequency vibrational spectroscopy," J. Chem. Phys. 144, 244711 (2016).

${ }^{35}$ Y. R. Shen, Fundamentals of Sum-Frequency Spectroscopy, Cambridge Molecular Science, 1st ed. (Cambridge University Press, Cambridge, UK, 2016).

${ }^{36}$ A. Morita, Theory of Sum Frequency Generation Spectroscopy, Lecture Notes in Chemistry, 1st ed. (Springer Singapore, Singapore, 2018).

${ }^{37}$ N. Bloembergen and P. S. Pershan, "Light waves at the boundary of nonlinear media," Phys. Rev. 128, 606-622 (1962).

${ }^{38}$ Y. R. Shen, "Basic theory of surface sum-frequency generation," J. Phys. Chem. C 116, 15505-15509 (2012).

${ }^{39}$ W.-T. Liu and Y. R. Shen, "Surface vibrational modes of $\alpha$-quartz(0001) probed by sum-frequency spectroscopy,” Phys. Rev. Lett. 101, 016101 (2008).

${ }^{40}$ Y. R. Shen, The Principles of Nonlinear Optics, Wiley Classics Library, Wiley Classics Library ed. (Wiley-Interscience, Hoboken, NJ, 2003).

${ }^{41}$ R. W. Boyd, Nonlinear Optics, 3rd ed. (Academic Press, 2008).

${ }^{42}$ I. Šimon and H. O. McMahon, "Study of the structure of quartz, cristobalite, and vitreous silica by reflection in infrared," J. Chem. Phys. 21, 23-30 (1953).

${ }^{43}$ P. T. T. Wong, D. J. Moffatt, and F. L. Baudais, "Crystalline quartz as an internal pressure calibrant for high-pressure infrared spectroscopy," Appl. Spectrosc. 39, 733-735 (1985)

${ }^{44} \mathrm{D}$. Krishnamurti, “The Raman spectrum of quartz and its interpretation," Proc. Natl. Acad. Sci. India 47, 276-291 (1958).

${ }^{45}$ S. Yamaguchi, K. Shiratori, A. Morita, and T. Tahara, "Electric quadrupole contribution to the nonresonant background of sum frequency generation at air/liquid interfaces," J. Chem. Phys. 134, 184705 (2011).

${ }^{46}$ R.-h. Zheng, W.-m. Wei, and Q. Shi, "Theoretical investigation of quadrupole contributions to surface sum-frequency vibrational spectroscopy," Phys. Chem. Chem. Phys. 17, 9068-9073 (2015).

${ }^{47}$ H. Held, A. I. Lvovsky, X. Wei, and Y. R. Shen, "Bulk contribution from isotropic media in surface sum-frequency generation," Phys. Rev. B 66, 205110 (2002).

${ }^{48}$ N. Ji and Y.-R. Shen, "A novel spectroscopic probe for molecular chirality," Chirality 18, 146-158 (2006).

${ }^{49} \mathrm{~K}$. Wolfrum and A. Laubereau, "Vibrational sum-frequency spectroscopy of an adsorbed monolayer of hexadecanol on water. Destructive interference of adjacent lines," Chem. Phys. Lett. 228, 83-88 (1994).

${ }^{50}$ R. J. Pressley, Handbook of Lasers (Chemical Rubber, Cleveland, OH, 1971). 